\title{
Pneumotórax fechado em tamanduá mirim (Tamandua tetradactyla)
}

\author{
Closed Pneumothorax in an Injured Southern Tamandua (Tamandua tetradactyla) \\ Ronaldo José Piccoli', Daniel Henrique Carvalho dos Santos², \\ Mariana Pavelski \& Anderson Luiz de Carvalho'
}

\begin{abstract}
Background: Pneumothorax is characterized by the accumulation of air in the pleural space, either due to trauma or secondary to other conditions. Typically, pneumothorax is correlated with blunt trauma of the pulmonary parenchyma or penetrating trauma of the thoracic cavity, such as on being trampled upon or bitten, respectively. The therapeutic approach of this condition is rarely described in wild animals; therefore, the present study aims to describe the clinical manifestations, diagnostic method, and therapeutic aproach in a specimen of Tamandua tetradactyla with closed pneumothorax received for emergency care after being hit by a vehicle.

Case: A southern tamandua (T. tetradactyla) was received in our hospital after being hit by a vehicle. The patient presented with a state of stupor, nystagmus, a restrictive respiratory pattern, and muffling on auscultation of respiratory and cardiac sounds in the left antimer. Simultaneously with the physical examination, venous access was established, pain control was intravenously performed and oxygen therapy was started. After stabilization, the patient underwent abdominal ultrasound (abdominal-focused assessment with sonography for trauma) and thoracic radiographs. The abdominal ultrasound confirmed the presence of a small amount of free fluid in the evaluated recesses, i.e., hepatodiaphragmatic, splenorenal, cystocholic, and hepatorenal, suggesting the need for periodic ultrasonographic monitoring associated with the clinical evaluation of the patient because of suspicion of active hemorrhage. The thoracic radiographic image (ventrodorsal recumbency) revealed increased pulmonary opacity due to lobar retraction, marked by an enlarged gap between the pulmonary lobes and thoracic wall, and the formation of a radiolucent area between both structures. The lateral recumbency evidenced the dorsal displacement of the cardiac apex in relation to the sternum. In addition to the thoracic alterations, a transverse fracture in the middle third of the diaphysis of the left fibula was identified by radiographic examination. After extensive hair clipping and skin antisepsis of the left hemithorax, thoracentesis (an adapted technique from that used in domestic animals) was performed with access via the 8th intercostal space in its most dorsal presentation using a 21-gauge Butterfly needle attached to a three-way medical valve and $20-\mathrm{mL}$ syringe. The procedure allowed $100 \mathrm{~mL}$ air to be drained until the negative pressure of the cavity was restored. At the end of the drainage, the patient showed a remarkable improvement in the respiratory pattern, and follow control radiographs showed full pulmonary expansion and no recurrence. The possibility of active abdominal hemorrhage was disregarded because ultrasound monitoring after $6 \mathrm{~h}$ revealed no change in the amount of free fluid in the suppressed recess.

Discussion: Pneumothorax is an important condition that can be detected in injured domestic and wild animals that are run over. The association between a detailed clinical evaluation and radiographic examination was essential for the diagnosis and initiation of the appropriate therapy, contributing to the prognosis of the patient. The adaptation of the thoracocentesis technique used in domestic animals proved to be suitable for the treatment of this Tamandua tetradactyla.
\end{abstract}

Keywords: wild animals, thoracocentesis, pneumothorax, southern tamandua.

Descritores: animais silvestres, toracocentese, pneumotórax, tamanduá-mirim. 


\section{INTRODUÇÃO}

Pneumotórax é uma condição clínica caracterizada pela presença e acúmulo de ar no espaço pleural, decorrente do estabelecimento de comunicação pleurocutânea, pleuropulmonar e/ou pleuro-esofágica [8]. O extravasamento do ar no espaço pleural impede a expansão adequada dos pulmões e limita o processo de ventilação e oxigenação $[3,18]$. Essa afecção pode ser classificada, de acordo com sua etiologia, em aberta, fechada ou hipertensiva [15]. Normalmente, o quadro de pneumotórax é correlato a traumas contusos do parênquima pulmonar ou penetrantes da cavidade torácica, como atropelamento e mordeduras, respectivamente [18]. A ocorrência dessa condição também pode estar ligada a neoplasias pulmonares, infecções e/ ou ainda a iatrogenias $[15,18]$. Apesar de bem descrita em animais domésticos como cães e gatos, ainda são poucos os relatos dessa alteração em animais silvestres.

O tamanduá-mirim (Tamandua tetradactyla) tem distribuição geográfica restrita ao continente sul americano, sendo encontrado em vários países além do Brasil [12]. A espécie apresenta risco de extinção pouco preocupante, contudo ameaças como a caça, atropelamentos, ataques de cães domésticos e a destruição de habitats naturais, são fatores que podem contribuir para que num futuro próximo haja um maior declínio de suas populações e até mesmo sua extinção em algumas regiões [12,14].

O presente trabalho objetiva relatar as manifestações clínicas, diagnóstico e abordagem terapêutica de pneumotórax fechado em um espécime de Tamandua tetradactyla recebido para atendimento emergencial, haja vista que casos de pneumotórax nessa espécie são pouco documentados na literatura, sendo encontrado apenas um breve relato [2].

\section{CASO}

Um exemplar adulto, macho, de Tamandua tetradactyla foi recebido para atendimento emergencial após ter sido atropelado no município de Mercedes/PR. No exame clínico o animal apresentava temperatura retal de $34^{\circ} \mathrm{C}$, frequência cardíaca de 132 batimentos por min, frequência respiratória de 12 movimentos por min com padrão respiratório restritivo e sons respiratórios abafados na ausculta do hemitórax esquerdo. Observou-se também mucosas hipocoradas, tempo de preenchimento capilar (TPC) de $2 \mathrm{~s}$, auscultação cardíaca abafada e pulso fraco na artéria femoral medial.
O paciente apresentava nível de consciência de estupor e presença de nistagmo horizontal. Na palpação do membro pélvico esquerdo, em região dos ossos tíbia e fíbula, foi constatada crepitação e mobilidade óssea, sugestiva de fratura. Foram também evidenciadas escoriações na pele da mão, cabeça (em região do osso frontal), e membro pélvico esquerdo (região média de osso tíbia e fíbula).

Concomitante ao exame físico, foi estabelecido o acesso venoso, seguido pela instituição de analgesia com morfina (Dimorf ${ }^{\circledR 1}, 0,5 \mathrm{mg} / \mathrm{kg}, \mathrm{IM}$ ), meloxicam 0,2\% (Maxicam $\left.{ }^{\circledR 2}, 0,2 \mathrm{mg} / \mathrm{kg}, \mathrm{IM}\right)$, além de instituição de oxigenoterapia (via máscara). Devido aos achados do exame físico, após estabilizado, o paciente foi encaminhado para o setor de Diagnóstico por Imagem para avaliação diagnóstica. Foi optado iniciar por avaliação AFAST (Abdominal Focused Assessment with Sonography for Trauma), adaptando a técnica utilizada em animais domésticos (cães e gatos), e na sequência efetuou-se a avaliação radiográfica do tórax com emprego de projeções laterais e ventrodorsal. Por apresentar estado de estupor não foi necessária a contenção química do paciente, sendo possível a realização de ambos os exames apenas com contenção física. Na avaliação ultrassonográfica AFAST foi possível constatar a presença de pequena quantidade de líquido livre nos recessos avaliados (hepatodiafragmático (HD), esplenorrenal (SR) Figura 1, cistocólico (CC) e hepatorrenal (HR)), foi sugerido possibilidade de hemorragia ativa e realizado acompanhamento ultrassonográfico (Sonoace R3) ${ }^{3}$ associado ao quadro geral do paciente. Na avaliação das radiografias torácicas foi observado, na exposição

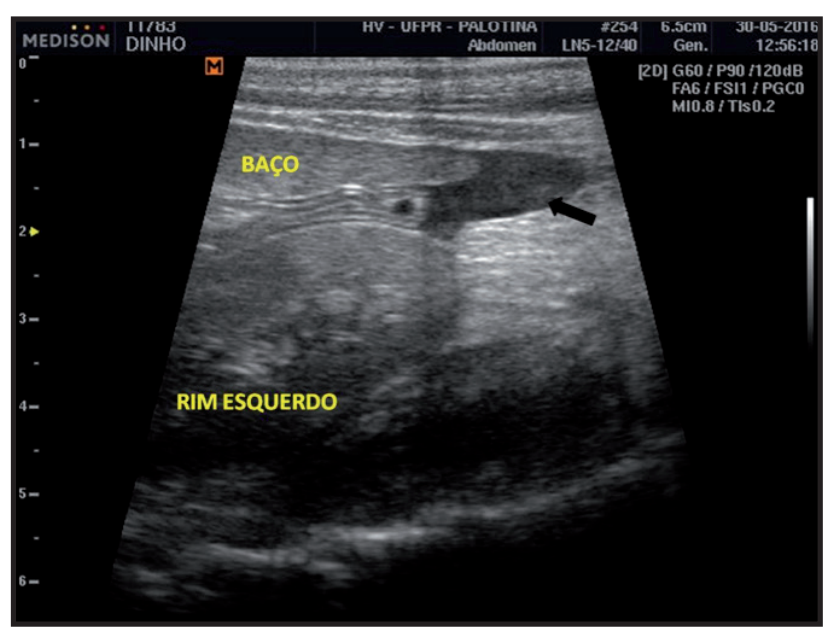

Figura 1. Imagem ultrassonográfica abdominal do paciente, com evidência de líquido livre (seta) em recesso esplenorrenal. 
ventrodorsal, a retração dos lobos pulmonares de todo o hemitórax esquerdo, marcada pelo distanciamento da parede torácica, com presença de área radiotransparente sugerindo acúmulo de ar em espaço pleural (Figura 2). Nas projeções laterais, foi observado o deslocamento dorsal do ápice cardíaco em relação ao esterno. Somada as alterações torácicas foi identificado ainda, por meio de exame radiográfico, a presença de fratura transversa em terço médio de diáfise do osso fíbula do antímero esquerdo.

Devido as alterações radiográficas e as manifestações clínicas foi definido diagnóstico de pneumotórax sendo indicado a manobra de toracocentese para drenagem, com adaptação da técnica descrita por Rozanski [17] para cães e gatos. Foi realizada ampla tricotomia da região torácica esquerda, seguida de antissepsia com iodopovidona degermante (Riodeine dermo suave degermante ${ }^{\circledR 4}$ ), álcool 70\% (RioGel Antisséptico ${ }^{\circledR 4}$ ) e iodopovidona tópico (Riodeine dermo suave tópico $\left.{ }^{\circledR 4}\right)$. Foi utilizado dispositivo scalp número 21 acoplado a uma torneira de três vias e uma seringa de $20 \mathrm{~mL}$, com os quais realizou-se a punção torácica

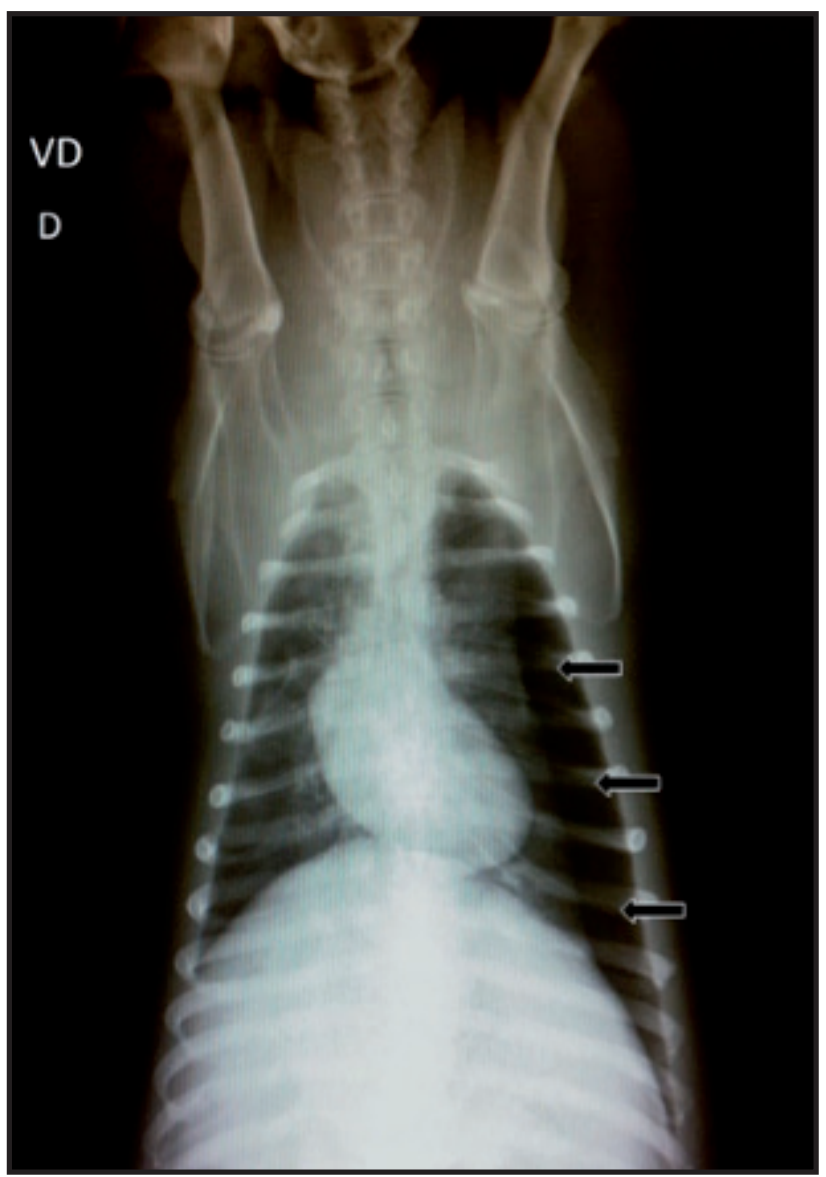

Figura 2. Imagem radiográfica torácica, em projeção ventrodorsal, com evidência de acúmulo de ar em hemitórax esquerdo (setas). no $8^{\circ}$ espaço intercostal esquerdo em seu ponto mais dorsal, pelo qual foram drenados aproximadamente $100 \mathrm{~mL}$ de ar. A retirada do scalp foi realizada após a constatação de ausência de ar livre no espaço pleural, pela formação de vácuo na seringa, que sinalizou a restituição da pressão negativa intratorácica. Ao término do procedimento, observou-se perceptível melhora no padrão respiratório do paciente, o qual foi submetido a estudo radiográfico controle. Na radiografia torácica foi possível observar ausência de espaço radiotransparente e plena expansão dos lobos pulmonares. O espécime foi mantido em observação e submetido a novo exame radiográfico $12 \mathrm{~h}$ após a drenagem do pneumotórax, no qual foi observado normalidade radiográfica. No exame ultrassonográfico controle não foi observado aumento na quantidade de líquido livre abdominal, o que associado a estabilidade dos parâmetros físicos descartou a possibilidade de hemorragia ativa.

O paciente não teve recidiva do pneumotórax e foi mantido no hospital veterinário para consolidação da fratura, e recebeu alta 42 dias depois admissão.

\section{DISCUSSÃO}

Os atropelamentos de fauna constituem um fator de impacto preocupante para todas as espécies silvestres, e apesar de estudos correlacionados aos atropelamentos de fauna serem bastante recentes no Brasil, sabe-se que muitas vezes as estatísticas obtidas são subestimadas [16,19].

As manifestações clínicas comumente presentes em casos de pneumotórax em cães e gatos são dispneia expiratória, taquipneia com padrão respiratório restritivo compensatório rápido e superficial, ansiedade ou estresse $[3,5,13]$. Todavia, outras alterações podem ser encontradas no exame físico desses animais, tais como: cianose, hipotensão ou hipertensão, aumento da ressonância na percussão do tórax e abafamento na ausculta dos sons cardíacos e respiratórios [3]. Parte das manifestações clínicas e achados de exame físico descritos na literatura foram constatadas no paciente atendido, como o padrão respiratório compensatório para melhorar a ventilação frente à dificuldade de expansão pulmonar, decorrente do extravasamento de ar na cavidade pleural $[3,5]$. No caso do paciente atendido, sugere-se que o ar foi oriundo de trauma contuso do parênquima pulmonar, já que não foi observada solução de continuidade entre o tórax e meio externo nem a existência de neoplasia e/ou infeção. Ressalta-se que o mecanismo de hiperventilação se estabelece na 
presença de ar no espaço pleural, porém ao decorrer da evolução do pneumotórax, esse mecanismo tornase ineficaz, tendo como consequência a elevação dos valores de $\mathrm{CO}_{2}$ arterial e condução do paciente a um quadro de acidose, que contribui para o óbito.

Os valores obtidos para temperatura retal, TPC, frequência cardíaca e frequência respiratória estavam dentro do intervalo fisiológico para a espécie [12]. Porém foi observado abafamento da ausculta pulmonar e cardíaca no antímero esquerdo. O estado de estresse/ansiedade não foi observado, devido ao quadro de estupor apresentado pelo paciente, que somado ao quadro de nistagmo horizontal corroboram ao possível quadro de trauma cranioencefálico (TCE) [20]. O quadro hipotensivo, a princípio, foi aferido por meio da palpação digital da artéria femoral medial, que se apresentou fraca/filiforme, porém não se utilizou um método paramétrico para determinação da pressão.

O uso de exames complementares tem colaborado muito para a expansão da medicina veterinária de animais silvestres, e possibilitado maior agilidade e acurácia no diagnóstico das afecções. As avaliações radiográficas de tórax são bastante rotineiras em animais atropelados [1]. Casos de pneumotórax em Tamandua tetradactyla são pouco documentados na literatura, assim como sua correção. Além do histórico e manifestações clínicas apresentadas pelo paciente, o exame de imagem atua como uma importante ferramenta para obtenção do diagnóstico de pneumotórax. As alterações observadas no exame radiográfico do paciente são compatíveis com as descritas na literatura $[5,21]$ para essa afecção. Cabe ressaltar que a depender do estado do paciente, o posicionamento para exposições radiográficas pode ser impossível/contraindicado, logo há necessidade de outra forma de diagnóstico auxiliar, a ultrassonografia torácica. Assim como o AFAST, o TFAST (Thoracic Focused Assessment with Sonography for Trauma) tem seu emprego para avaliação torácica em situações de traumas. Esse método de avaliação ultrassonográfica das condições torácicas já tem seu uso consolidado na medicina humana e vem se estabelecendo na medicina de animais domésticos (cães e gatos), com bons resultados na identificação de vários quadros como, por exemplo, pneumotórax e efusões $[4,9,17]$. Esta técnica de exame, baseada em uma rápida avaliação torácica, permite a triagem de pacientes com complicações respiratórias [4,9]. Para realização do mesmo não há necessidade de tricotomia, além de ser possível a sua execução com o animal em estação ou decúbito esternal $[4,9]$. Neste caso seria observado ausência de sinal de deslizamento pleural e artefatos em cauda de cometa, como descrito por [4], corroborando com as alterações radiográficas encontradas. Apesar de efetivo, a técnica de TFAST não foi realizada no paciente.

Semelhante ao observado no exame ultrassonográfico deste espécime, a presença de líquido livre abdominal já foi descrito como um achado ultrassonográfico normal [10] em tamanduás-bandeira (Myrmecophaga tridactyla), contudo não foram encontradas informações especificas desta condição para espécimes de Tamandua tetradactyla.

Com relação a técnica empregada para correção do pneumotórax, adaptaram-se as técnicas já descritas para cães e gatos, frente a ausência de descrições detalhadas sobre o uso de toracocentese em tamanduás. Porém atenta-se que esses indivíduos apresentam particularidades anatômicas que devem ser pontuadas, como a presença de um tórax mais alongado que cães e gatos, e por possuírem 17 vertebras torácicas e 17 costelas [11]. Não foram encontrados trabalhos que descrevessem a anatomia pulmonar de tamanduá-mirim, todavia, de acordo com um trabalho [6], para tamanduá-bandeira (Mymercophaga tridactyla) a lobação pulmonar apresentou-se semelhante à descrita para cães [7], a saber: quatro lobos no pulmão direito (cranial, médio, caudal e acessório) e dois no pulmão esquerdo (cranial e caudal).

O pneumotórax é uma afecção importante que pode ser identificada em animais silvestres vitimados por atropelamento, o diagnóstico precoce e a instituição da terapêutica adequada são decisivos no prognóstico do paciente. A associação da avaliação clínica minuciosa e do exame radiográfico são fundamentais para realização do diagnóstico. A adaptação da técnica de toracocentese utilizada em animais domésticos foi utilizada com sucesso no Tamandua tetradactyla relatado e o uso da mesma pode ser adequado à outras espécies, desde que consideradas as especificidades anatômicas destas.

\section{MANUFACTURERS}

${ }^{1}$ Laboratório Cristália. Itapira, SP, Brazil.

${ }^{2}$ Ouro Fino Saúde Animal. Cravinhos, SP, Brazil.

${ }^{3}$ Samsung Medison Co. Seoul, South Korea.

${ }^{4}$ Rioquímica Indústria Farmacêutica. São José do Rio Preto, SP, Brazil. 


\section{REFERENCES}

1 Alves L.S., Charlier M.G.S., Shigue D.A., Vulcano L.C. \& Teixeira C.R. 2014. Radiografia, tomogradia e reconstrução tridimensional de fratura lombar salter-harris tipo i em tamanduá-bandeira (Myrmecophaga tridactyla). In: Congresso Nordestino de Especialidades Veterinárias de Pequenos Animais - CONEVEPA (Recife, Brazil). 1 CD ROM.

2 Alves L.S., Shigue D.A., Vulcano L.C. \& Teixeira C.R. 2014. Contribuição radiográfica no diagnóstico de peneumotórax em Tamanduá-mirim (Tamandua tetradactyla). In: Congresso Nordestino de Especialidades Veterinárias de Pequenos Animais - CONEVEPA (Recife, Brazil). 1 CD ROM.

3 Arruda I.V. 2011. Pneumotórax: Revisão de Literatura. 32f. São Paulo, SP. Monografia (Especialização) - Curso de Medicina Veterinária, Medicina Veterinária Intensiva de Pequenos Animais, Universidade Federal Rural do Semi Árido.

4 Boysen S.R. \& Lisciandro G.R. 2013. The Use of Ultrasound for Dogs and Cats in the Emergency Room. Veterinary Clinics of North America: Small Animal Practice. 43(4): 773-797.

5 Fossum T.W. 2008. Cirurgia do Sistema Respiratório Inferior: cavidade pleural e diafragma. In: Fossum T.W. (Ed). Cirurgia de pequenos Animais. 3.ed. Rio de Janeiro: Elsevier, pp.896-929.

6 Giraldi A.C.C., Aires L.P.N., Rodrigues R.F., Cruvinel T.M.A \& Melo A.P.F. 2016. Anatomia e Segmentação Pulmonar de Tamanduá-Bandeira (Mymercophaga tridactyla - Linnaeus, 1758) de vida livre. Arquivo Brasileiro de Medicina Veterinária e Zootecnia. 69(1): 22-28.

7 Hare W.C.D. 1986. Sistema Respiratório do Carnívoro. In: Getty R. (Ed). Sisson/Grossman: Anatomia dos Animais Domésticos. 5.ed. Rio de Janeiro: Guanabara Koogan, pp.1465-1480.

8 Hawkins E.C. 2015. Clinical Manifestations of the Pleural Cavity and Mediastinal Disease. In: Nelson R.W. \& Couto C.G. (Eds). Small Animal Internal Medicine. 5th edn. St. Louis: Elsevier, pp.323-328.

9 Lisciandro G.R. 2014. The Vet Blue Lung Scan. In: Lisciandro G.R. (Ed). Focused Ultrasound Techniques for the Small Animal Practitioner. Ames: Wiley Blackwell, pp.166-188.

10 Lopes É.R., Morgado T.O., Meireles Y.S., Jorge A.A., Zago A.A.Q., Corrêa S.H.R., Paz R.C.R da \& Néspoli P.B. 2015. Ultrassonografia abdominal de tamanduás-bandeira (Myrmecophaga tridactyla Linnaeus, 1758) mantidos em cativeiro. Pesquisa Veterinária Brasileira. 35(11): 919-924.

11 Macedo B.C., Lima A.R., Pereira L.C. \& Branco É. 2013. Descrição morfológica dos ramos colaterais da aorta abdominal do tamanduá-mirim (Tamandua tetradactyla). Biotemas. 26(1): 173-180.

12 Miranda F. 2012. Manutenção de Tamanduás em Cativeiro. São Carlos: Cubo, 302p.

13 Moraillon R., Legeay Y. Boussarie D. \& Sénécat O. 2013. Manual Elsevier de Veterinária: diagnóstico e tratamento de cães, gatos e animais exóticos. 7.ed. Rio de Janeiro: Elsevier, 916p.

14 Ortunho V.V., Souza L.O., Santos L. \& Antonietti N. 2014. Case of treatment of fracture of tibia and fibula female anteater (Anteater tetradactyla). Revista Brasileira de Higiene e Sanidade Animal. 8(3): 130-137.

15 Puerto D.A. \& Waddell L.S. 2016. Pleural Space Disease: Pneumothorax. In: Aronson L.R. (Ed). Small Animal Surgical Emergencies. Oxford: Wiley-Blackwell, pp.327-336.

16 Rezini J.A. 2010. Atropelamento de mamíferos em rodovias do leste dos estados do Paraná e Santa Catarina, Sul do Brasil. 50f. Curitiba, PR. Dissertação (Mestrado) - Curso de Ciências Biológicas, Universidade Federal do Paraná.

17 Rozanski E. 2016. Thoracic Trauma. In: Ettinger S.J., Feldman E.C. \& Côté E. (Eds). Textbook of Veterinary Internal Medicine. 8th edn. St. Louis: Saunders, pp. 1641-1649.

18 Rozanski E.A. \& Rush J. 2013. Respiratory emergencies: Pneumothorax. In: Rozanski E.A. \& Rush J. (Eds). Small Animal Emergency and Critical Care Medicine. London: Manson Publishing, pp.75-76.

19 Sássi C.M., Nascimento A.A.T., Miranda R.F.P. \& Carvalho G.D. 2013. Levantamento de animais silvestres atropelados em trecho da rodovia BR482. Arquivo Brasileiro de Medicina Veterinária e Zootecnia. 65(6): 1883-1886.

20 Siqueira E.G.M., Rahal S.C., Vassalo F.G., Araújo F.A.P. \& Agostinho F.S. 2013. Trauma Cranioencefálico em Pequenos Animais. Veterinária e Zootecnia. 20(1): 112-123.

21 Thrall D.E. 2010. O espaço pleural. In: Thall D.E. (Ed). Diagnóstico de Radiologia Veterinária. 5.ed. Rio de Janeiro: Elsevier, pp.555-567. 